\title{
Determination of Renal Changes by Ultrasonography in Patients with Type-2 Diabetes Mellitus
}

\author{
Nosheen Arshad $^{1^{*}} \quad$ Muhammad Rohail Amjad $^{1} \quad$ Saba Ajmal $^{1} \quad$ Muhammad Talha Zia $^{1} \quad$ Amir Ali $^{1}$ \\ Tayyaba Sakhawat ${ }^{1}$ Haris Ahmad Ameen ${ }^{1}$ Hassan Raza ${ }^{1} \quad$ Homaina Ijaz $^{1} \quad$ Fahmeeda Ansari $^{2}$ \\ S. Muhammad Yousaf farooq ${ }^{2}$. \\ 1.Medical Imaging Doctor, University Institute of Radiological Sciences and Medical Imaging Technology, \\ University of Lahore, Lahore-Pakistan \\ 2.University Institute of Radiological Sciences and Medical Imaging Technology, University of Lahore, Lahore- \\ Pakistan
}

\begin{abstract}
Background: Diabetes Type 2 causes damage to the kidneys; leading to diabetic nephropathy and high blood pressure. The aim of this study is determination of renal changes on ultrasonography in diabetic patients. Objective: This study evaluates changes in kidneys in patients presenting with type II diabetes mellitus having normal renal function test as compared to non-diabetics. Methods: It was a cross sectional descriptive study conducted on 116 patients with type 2 diabetes mellitus by using convenient sampling technique. The study was conducted at Ultrasound Department of Gulab Devi hospital Hospital from July 2019 to September 2019. Results: Out of 116 patients there were $43(37.1 \%)$ female and $73(62.1 \%)$ were male. The mean age of the participants was 53.24 \pm 10.49 . This study shows that the mean volume of Rt kidney was $1.229 \mathrm{E} 2 \pm 38.39$ ranging from $25 \mathrm{ml}$ to $218 \mathrm{ml}$ and mean volume of Lt kidney was $1.1691 \mathrm{E} 2 \pm 41.96$ ranging from $26 \mathrm{ml}$ to $231 \mathrm{ml}$ in patients presenting with DM Type 2 and mean parenchymal thickness of the Rt kidney $14.40 \pm 6$ range from $0.86 \mathrm{~mm}$ to $1.25 \mathrm{~mm}$ and Lt kidney $13.7 \pm 5$ range from $1.2 \mathrm{~mm}$ to $25 \mathrm{~mm}$. This study showed that mean volume of both kidneys and parenchymal thickness increased in patients having diabetes mellitus. Conclusion: Ultrasound is a reliable and easily available modality to detect renal changes and complications in earlier stages of Diabetes mellitus.
\end{abstract}

Keywords: Type II diabetes mellitus, Renal changes, Ultrasonography

DOI: $10.7176 / \mathrm{JHMN} / 68-07$

Publication date: November $30^{\text {th }} 2019$

\section{Introduction}

Kidneys are bean shaped structures located on sides of T12-L3 vertebrae ${ }^{1}$. One of the important organs that are affected by diabetes are kidneys. Most common cause of end stage renal disease is diabetes in most parts of the world. Involvement of kidney, directly and indirectly increases the involvement of other organs and increases mortality and morbidity in diabetic patients. Type-2 diabetes prevalence has increased in Pakistan due to increase in industrialization of societies, immobility and life style. ${ }^{2}$ Diabetes mellitus is a diseases caused by disturbed, fat and protein metabolism. It is mainly characterized by hyperglycemia ${ }^{3}$.Diabetes mellitus has two types Type I and Type II diabetes.. Metabolism of all foodstuffs is changed in both types of diabetes mellitus. Insulin resistance and less insulin uptake has a prominent effect on body organs and causes increase in glucose uptake, hence glucose concentration increases in body. ${ }^{4}$.

Signs and symptoms of diabetes are ignored by many because of chronic progression of the disease. People do not consider this disease as a serious problem so the effects of hyperglycemia are not demonstrated immediately. Diabetes can start damage a few years before symptoms become observable. Considering the asymptomatic nature of type 2 Diabetes, warning signs are unexplained weight reduction, tiredness, irritability, dry mouth, itching, feet numbness, reactive hypoglycemia and eyesight weakness ${ }^{5}$. One of the complications of diabetes is diabetic nephropathy. It refers to functional and structural kidney abnormalities in diabetic patients. Structural abnormalities include tubular atrophy, thickness of glomerular basement membrane increased, kidneys hypertrophy, and interstitial fibrosis. On the other hand functional abnormalities include systematic hypertension, and loss of renal function ${ }^{3}$.

Approximately 285 million people have diabetes mellitus, between the age of 20 to 79 years $^{6}$. In low- and middle- income countries more than $80 \%$ deaths occur due to diabetes mellitus. Diabetes mellitus increases the risk of stroke and heart diseases. $50 \%$ of diabetic people die because of cardiovascular diseases.

Due to its many advantages, ultrasound is greatly used in evaluation of mostly kidney related disease. Has been routinely used for decades in the diagnosis of different renal diseases. Following changes are seen while doing renal ultrasound of diabetic patients: It is seen that renal changes due to diabetes mellitus are easily seen on ultrasonography. Ultrasound is used also to exclude non diabetes - related renal disorders e.g. renal stones, masses or hydronephrosis ${ }^{7}$. Ultrasound is a useful investigating tool for the evaluation of renal diseases due to ease of performance, with the advantage of affordability, easy availability, low cost and not having ionizing radiations. Radiologists and nephrologists also used this modality to assess the renal function ${ }^{8}$. 
The rationale behind this research is to enhance the use of ultrasound in diagnosing diabetes mellitus type-2 by looking at the structural changes taking place in the kidney of a diabetes mellitus -2 patients.

\section{Methods}

It was a cross sectional descriptive study conducted on 116 patients suffering from diabetes mellitus type 2 by using convenient sampling technique. Study was conducted at the ultrasound department of Gulab Devi Hospital from July 2019 to September 2019. The scanning of renal parenchyma was performed with Canon Apiol 300 ultrasound machine and using a convex probe with frequency of 3.5 to $5 \mathrm{MHz}$. Observation was made on gray scale ultrasound machine. The study was conducted to find out the effects on parenchymal thickness of kidneys , kidney volume and kidney size in patients with Type 2 Diabetes mellitus. SPSS 20 was used for statistical, frequency and percentages were found.

\section{Results}

It was a cross sectional descriptive study that was conducted in the ultrasound department of Gulab Devi Hospital Lahore that included 116 patients. The data was collected by using consecutive technique from July 2019 to September 2019. A questionnaire was used for each patient, which was filled out on the basis of diabetic history and ultrasound image findings.

Out of 116 patients there was 43(37.1\%) female and 73(62.1\%) were male.(Table no.1).Patient of all ages included in research.. Total 116 patients were included in the research.. Mean age of the patients was 53.24 \pm 10.49 . The minimum age was 21 years and maximum was 83 years. There was no gender specification (Table no.2).

According to the descriptive study (Table 3.1) shows mean volume of Rt kidney was $1.229 \mathrm{E} 2 \pm 38.39$ ranging from 25 to 218 and (Table 3.2) shows the mean volume of Lt kidney $1.1691 \mathrm{E} 2 \pm 41.96$ ranging from 26 to 231 in patients presenting with DM Type 2. Table 4.1 shows mean parenchymal thickness of the Rt kidney $14.40 \pm 6$ range from $0.86 \mathrm{~mm}$ to $1.25 \mathrm{~mm}$ and Lt kidney $13.7 \pm 5$ range from $1.2 \mathrm{~mm}$ to $25 \mathrm{~mm}$.

\begin{tabular}{|l|l|r|r|r|c|}
\hline \multicolumn{2}{|c|}{} & \multicolumn{2}{|c|}{ Gender } \\
\hline \multirow{2}{*}{ Valid } & Frequency & Percent & Valid Percent & Cumulative Percent \\
\cline { 2 - 7 } & M & 43 & 37.1 & 37.1 & 37.1 \\
\cline { 2 - 7 } & Total & 73 & 62.9 & 62.9 & 100.0 \\
\hline
\end{tabular}

Table no:1 gender distribution

\begin{tabular}{|l|r|r|r|r|c|}
\hline \multicolumn{7}{|c|}{ Age } & \multicolumn{1}{c|}{ Mean } & Std. Deviation \\
\hline Age & $\mathrm{N}$ & Minimum & Maximum & \multicolumn{1}{c|}{ Mean } \\
\hline Valid N (list wise) & 116 & 21.00 & 83.00 & 53.2414 & 10.49689 \\
\hline
\end{tabular}

Table no: 2 Age distribution

\begin{tabular}{|l|r|r|r|r|c|}
\hline & Right Kidney Vol. \\
\hline Right KidneyVol & N & Minimum & Maximum & Mean & Std. Deviation \\
\hline Valid N (list wise) & 116 & 25.00 & 218.00 & $1.2292 \mathrm{E} 2$ & 38.39487 \\
\hline
\end{tabular}

Table 3.1 shows Rt kidney volume.

\begin{tabular}{|l|c|c|c|c|c|}
\hline \multicolumn{7}{|c|}{ Left Kidney Vol. } & Mean & Std. Deviation \\
\hline LeftKidneyVol & $\mathrm{N}$ & Minimum & Maximum & Mean & 41.90769 \\
\hline Valid N (list wise) & 116 & 26.00 & 231.00 & $1.1691 \mathrm{E} 2$ & \\
\hline
\end{tabular}

Table 3.2 shows Lt kidney volume

\begin{tabular}{|l|r|r|r|r|c|}
\hline \multicolumn{7}{|c|}{ Right Kidney Parenchymal Thickness } \\
\hline & $\mathrm{N}$ & \multicolumn{1}{|c|}{ Minimum } & Maximum & \multicolumn{1}{c|}{ Mean } & Std. Deviation \\
\hline RightKidneyParenchymal & 116 & .86 & 25.00 & 14.4057 & 6.32369 \\
\hline Valid N (list wise) & 116 & & & & \\
\hline
\end{tabular}

Table 4.1 shows Rt kidney parenchymal thickness. 


\begin{tabular}{|l|c|r|r|r|c|}
\hline \multicolumn{7}{|c|}{ Left Kidney Parenchymal Thickness } \\
\hline & $\mathrm{N}$ & Minimum & Maximum & Mean & Std. Deviation \\
\hline LeftKidneyParenchymal & 116 & 1.20 & 25.00 & 13.3793 & 5.89602 \\
\hline Valid N (list wise) & 116 & & & & \\
\hline
\end{tabular}

Table 4.2 shows Lt kidney parenchymal thickness.

Static analysis showed that mean volume of both kidneys and parenchymal thickness (although no significant corticomedullary difference) increased in patients presenting with Diabetes mellitus II with normal renal function. Scans

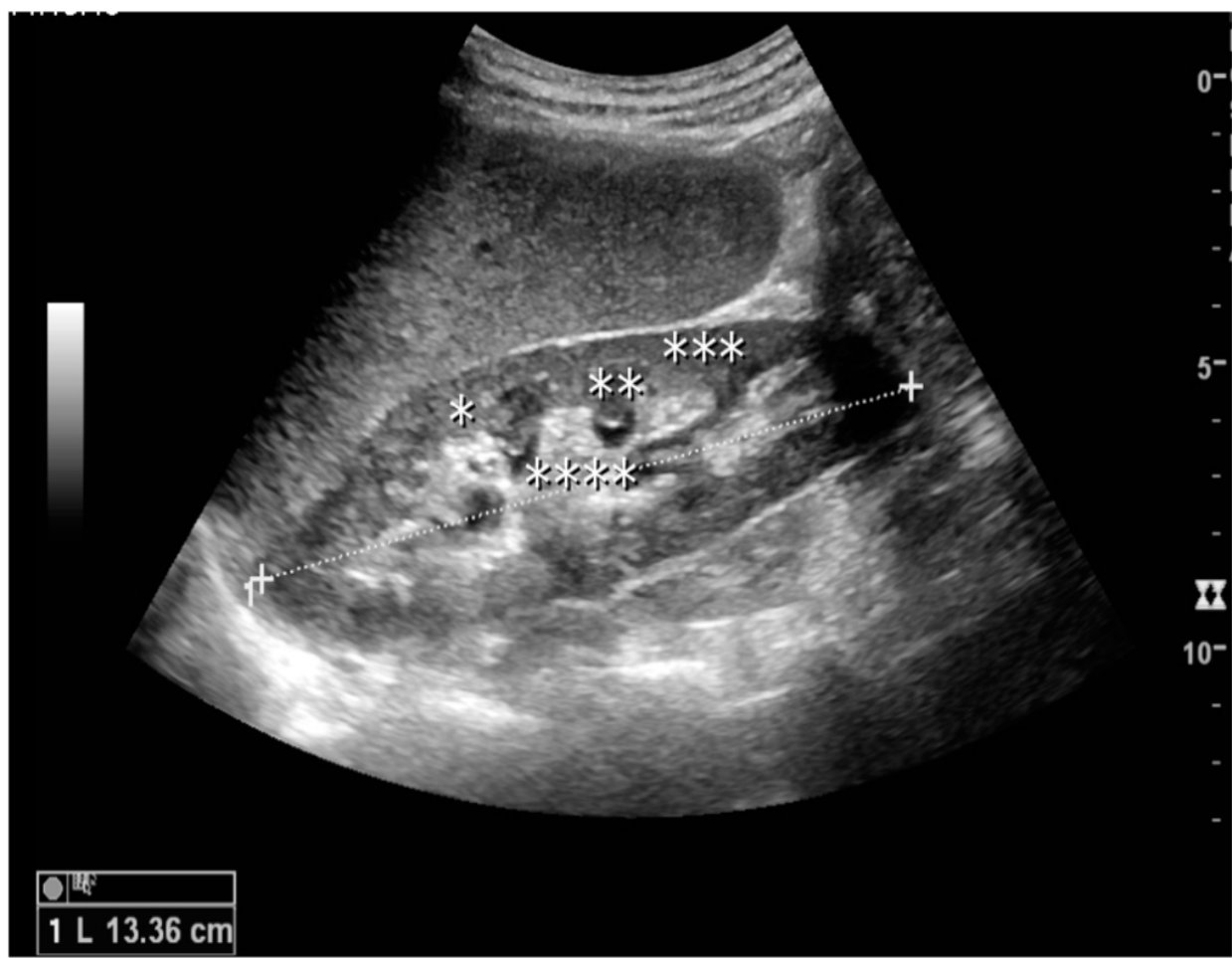

Figure 1: Normal left kidney is shown on Ultrasound with normal values.

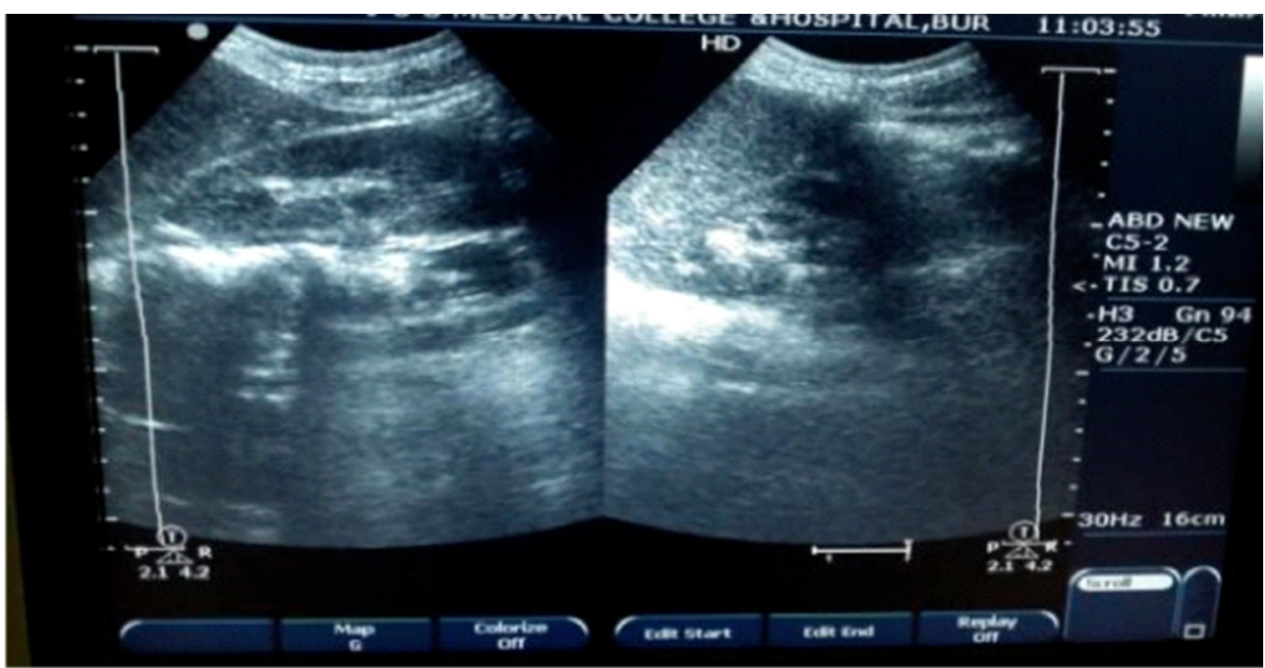

Figure 2: Shows increased echogenicity and increased parenchaymal thickness 


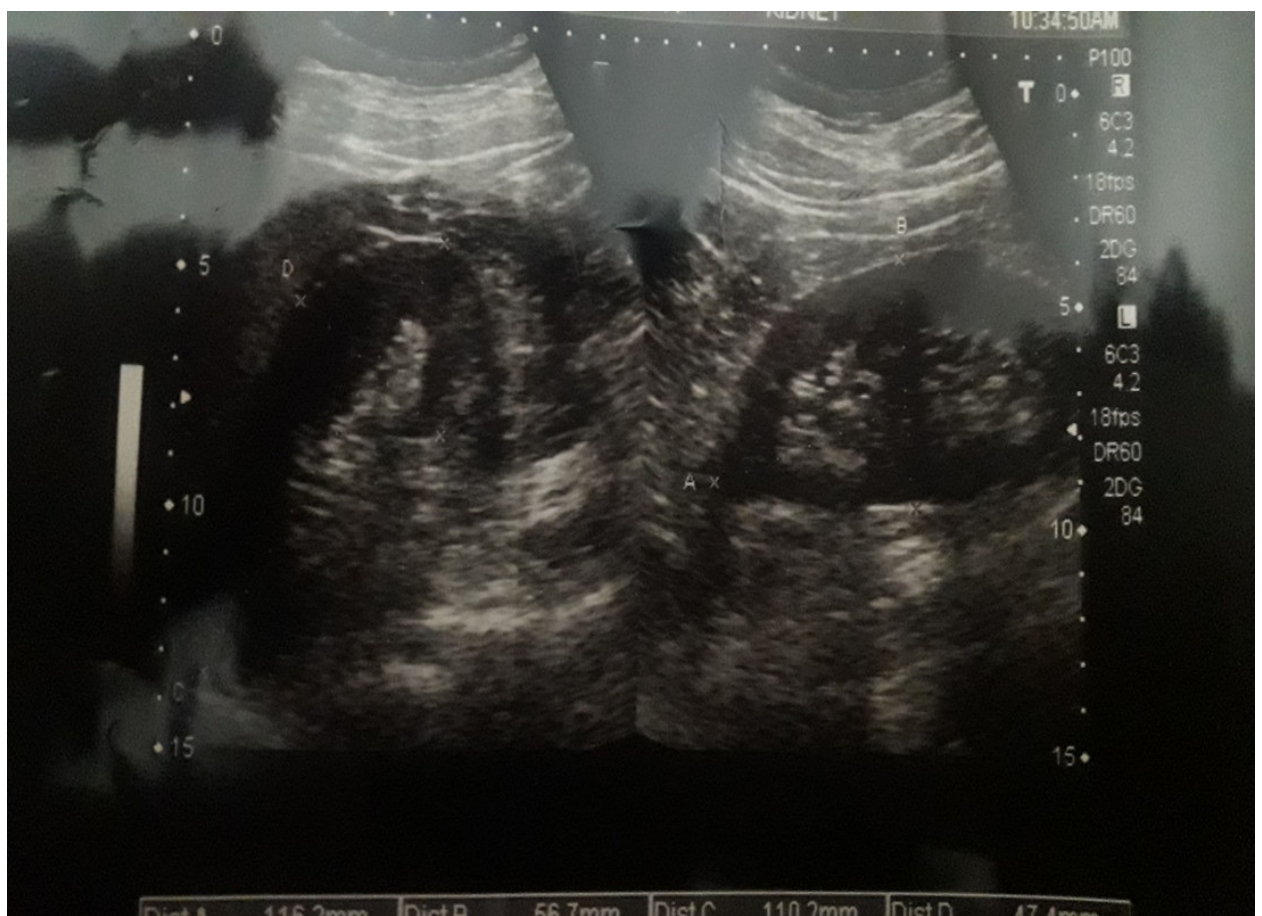

Fig no 3: shows mild increased volume.. increased parenchymal thickness with normal renal function..

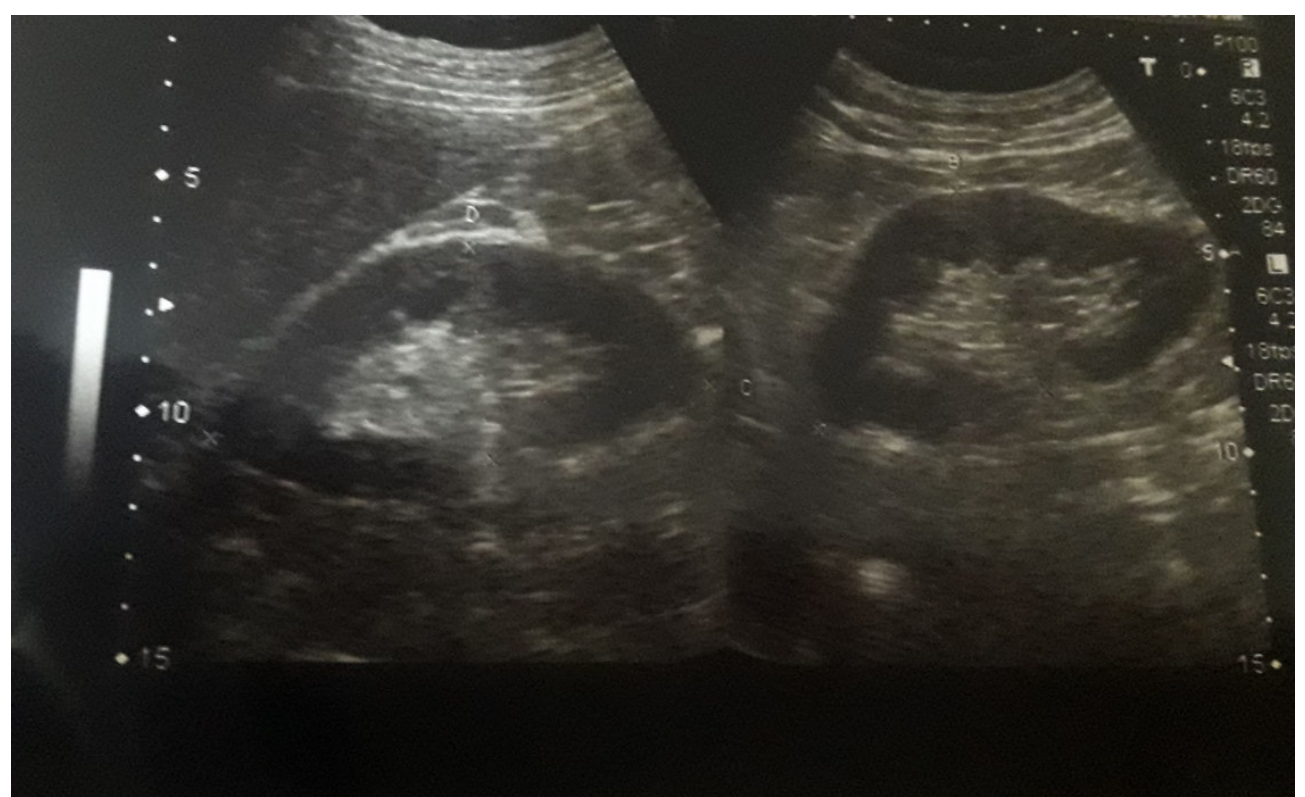

Fig no 4: mild increased volume with normal renal function. 


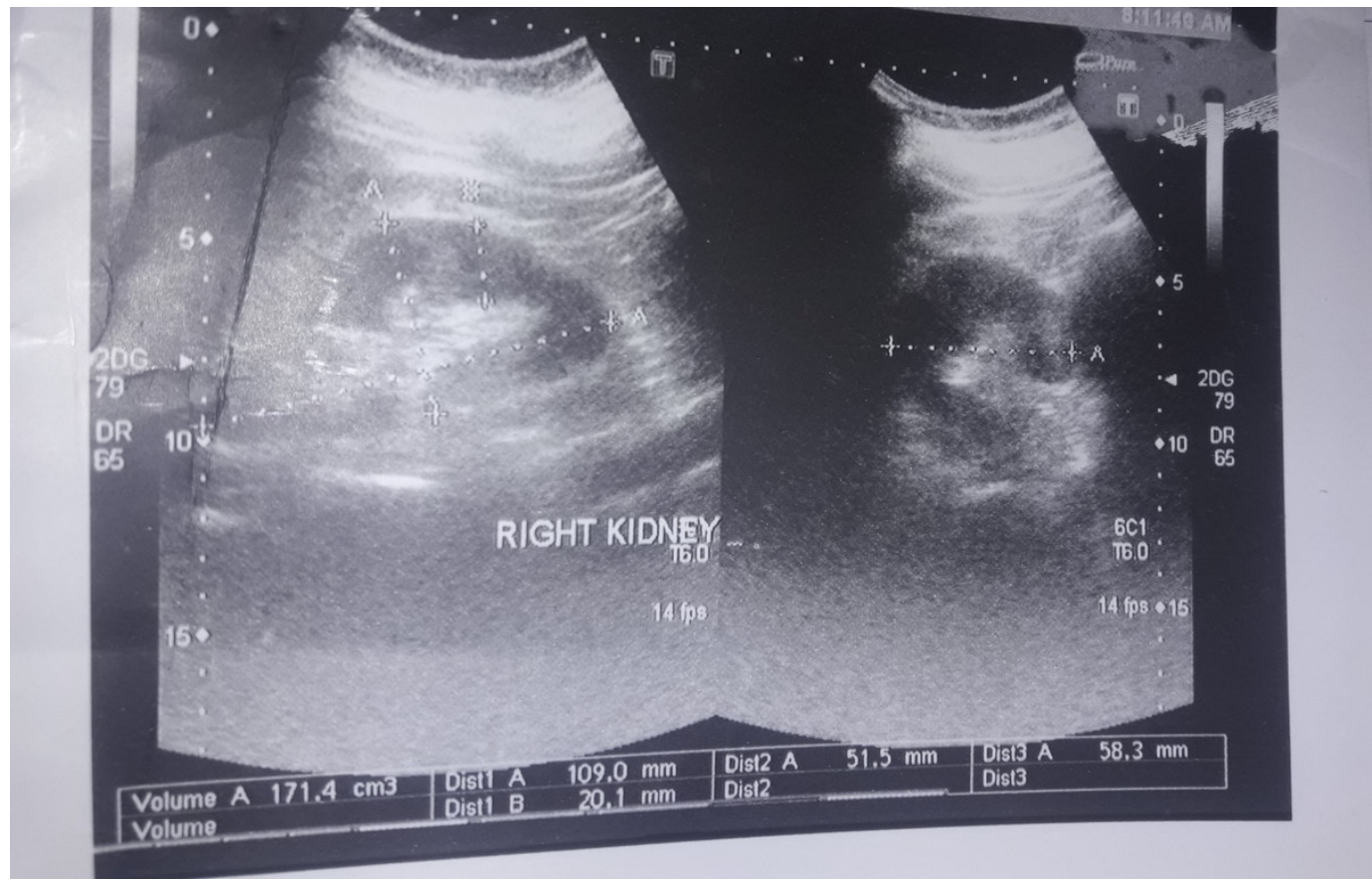

Fig no 5: both volume and parenchymal thickness are increased.

\section{Discussion}

It was cross-sectional study, conducted in the Radiology Department of Gulab Devi Chest Hospital, Lahore that included 116 patients. The data was collected using consecutive technique from July 2019 to September 2019. Diabetes mellitus results abnormality in insulin production and due to its effect. It is a destructive disease which ultimately effects almost all the organs of the body. It has many consequences but most common is diabetic nephropathy. It is not very common in children and younger patients, mostly seen in older patients. Gray scale Ultrasound is the first choice to detect renal changes caused by diabetes mellitus II. Routinely ultrasonography of diabetic patients with normal renal function can help to detect several renal changes such as increase in renal volume and parenchymal thickness.

This study showed that Out of 116 patients there was 43(37.1\%) female and 73(62.1\%) was male. Mean age of the patients was $53.24 \pm 10.49$. According to this study mean volume and mean parenchymal thickness increased in diabetic patients. This study is in total agreement with the study presented by Joseph Dlama Zira Et al (2017) Department of Radiology, Nigeria. For the assessment of kidneys ultrasonography 119 cases of diabetes were compared with 101 non diabetic. Increase in parenchymal thickness, volume and renal width in Diabetic patients was seen. Therefore the ultrasound is helpful to provide information for the detection of renal changes with Diabetes Mellitus Type-2 patients?

The result of this study is also a confirmation with the study of Mohammad A. Ali Omar et al, carried out in 2014 on "Ultrasound of diabetic patients "conducted on 150 patients which concluded increase in size of right and left kidneys as well as their echogenicity respectively on ultrasound ${ }^{10}$.

This study has similar results to a study conducted by Vinayaka Undemane Shivashankara, Siddharudha Shivalli in 2014 that included 70 patients, Out of which $67.1 \%$ were male and $32.9 \%$ were female. This comparative study evaluated that sonographic findings of renal changes are reliable to assess the severity of disease ${ }^{11}$.

The result of this study showed small difference from the study done by SaddigD. Jastaniah, et al (2013) "Diagnosis of kidney abnormalities in type -2 diabetic patient using ultrasound. This study was conducted on 400 patients aged 13-93 years with Type-2 diabetes mellitus. Most patients had grade I nephropathy detected on ultrasonography, other had normal renal size and echogenicity.

\section{Conclusion}

It was concluded that there was significant increase in mean volume and parenchymal thickness of both kidneys with normal renal function test. Therefore, Gray scale Ultrasound is a reliable and easily available modality to detect renal changes and complications in Diabetic Patients.

\section{References}

Standaring SU, Gray's Anatomy, $2008 ; 39^{\text {th }}$ edition. 1099. 
Shahbazian H, Rezaii I. Diabetic kidney disease; review of the current knowledge. Journal of renal injury prevention. 2013;2(2):73.

Zira JD. Sonographic Assessment of Renal Sizes, Parenchymal Thickness and Volume in Patients With Type-2 Diabetes Mellitus. PJR. 2017;27(3).

Omer MAA, Eljack AH, Gar-alnabi ME, Mahmoud MZ, Elseid M, Edam GA. Ultrasonographic Characteristics of Diabetes Impacts in Kidneys' Morphology. Open Journal of Radiology. 2014;4(04):301.

Ramachandran A. Know the signs and symptoms of diabetes. The Indian journal of medical research. 2014;140(5):579.1. Pecoits-Filho R, Abensur H, Betônico CC, Machado AD, Parente EB, Queiroz M, et al. Interactions between kidney disease and diabetes: dangerous liaisons. Diabetology \& metabolic syndrome. 2016;8(1):50.

Pecoits-Filho R, Abensur H, Betônico CC, Machado AD, Parente EB, Queiroz M, et al. Interactions between kidney disease and diabetes: dangerous liaisons. Diabetology \& metabolic syndrome. 2016;8(1):50.

Saddig D. Jastaniah NMA, Ibrahim A. Awad, Hashim R. Fida, Hammad H. Elniel. Evaluation of Renal Disorders in Type 2 Diabetic Patients Using Ultrasonography. published by Open Journal of Medical Imaging. 2013;3:165-70.

Shivashankara VU, Shivalli S, Pai BS, Acharya KD, Gopalakrishnan R, Srikanth V, et al. A comparative study of sonographic grading of renal parenchymal changes and estimated glomerular filtration rate (eGFR) using Modified Diet in Renal Disease formula. Journal of clinical and diagnostic research: JCDR. 2016;10(2):TC09.

Aldukhayel A. Prevalence of diabetic nephropathy among Type 2 diabetic patients in some of the Arab countries. International journal of health sciences. 2017;11(1):1.

S. Nayak1 SKT, S. Das1, B. P. Das2, C. R. Kar3. Evaluation of type of nephropathy in patients with type -2 diabetes mellitus. Journal of Diabetes Mellitus. 2017;7:281-93.

Shaw M, Bhagat S, Panda B, Nisa S, Das B, Panda A. Diabetic Nephropathy: Ultrasound, Color Doppler and Biochemical Correlation-A 2 Year Study. 